\title{
Acid-Base Properties of Porphyrins in Nonaqueous Solutions
}

\author{
Vladimir G. Andrianov, ${ }^{@}$ and Ol'ga V. Malkova \\ Ivanovo State University of Chemistry and Technology, Ivanovo, 153000, Russia \\ ${ }^{\circledR}$ Corresponding authorE-mail: shukhto@isuct.ru
}

\begin{abstract}
The study of acid-base properties of porphyrins have been started in our university under the guide of our teacher Professor B. D. Berezin in the beginning of the $60^{\text {th }}$. The obtained results can be considered as a significant contribution to the porphyrin chemistry. In proton-donor media porphyrins can be stepwise protonated forming the mono-and dications and deprotonated forming mono- and dianions. Acid-base forms of porphyrins can be stabilized by electronic, solvation and steric factors ${ }^{[8-9]}$ Electronic factors are characterised by changing of electronic density in the porphyrin macroring during protonation or deprotonation. Substituents in $\beta$-, meso-positions and at inner $N$-atoms play an important role in the redistrubution of the electron density. Stabilization of ionic forms in solution occur by solvation with the solvent molecules and by association with $M^{+}$or $X^{-}$. Ability of the macrocycle to deformation is an important steric factor. Reaction with inorganic base in the polar solvent leads to formation of the ion-molecular associate $\mathrm{N}-\mathrm{H}^{\cdots} \mathrm{OH} \mathrm{I}^{\left[{ }^{6]}\right.}$ Complete ionization of the $N$-Hbond may occur only in non-aqueous media in tetraalkylammonium hydroxides or at high concentration of alkoxide-anions. ${ }^{[10-11]}$ The negative charge of the monoanionic forms is delocalized in the coordination center and concentration of ionic forms is decreased in the order $I V>V>V I .{ }^{[12]}$ Parameters of the acidic dissociation of the porphyrins in methanol obtained by method of solubility are listed in Table 1 . The value of pK $K_{3}$ is increased in the order: $\mathrm{H}_{2} \mathrm{TPP}\left(\mathrm{p}-\mathrm{OCH}_{3}\right)<\mathrm{H}_{2} \mathrm{TPP}<\mathrm{H}_{2} \mathrm{DP}<\mathrm{H}_{2} \mathrm{PP}<\mathrm{H}_{2} \mathrm{EP}<\mathrm{H}_{2} \mathrm{MP}<\mathrm{H}_{2} \mathrm{Chl}(\mathrm{a})<\mathrm{H}_{2} \mathrm{Chl}(\mathrm{b})$. Acid ionization of porphyrins in DMSO was studied by spectropotentiometric titration with $\left[\mathrm{N}\left(\mathrm{CH}_{3}\right)_{4}\right] \mathrm{OH}$. The stability of monoanions in DMSO is increased in the order: $\mathrm{H}_{2} \mathrm{P}<\mathrm{H}_{2} \mathrm{TBP}<<\mathrm{H}_{2} \mathrm{TAP}<\mathrm{H}_{2} \mathrm{Pc}\left(\mathrm{SO}_{3}^{-}\right)$. The nature of the solvent have influence on acid properties of the porphyrins. The solvent can stabilise neutral and ionic forms of porphyrin. The monoanion is stabilised in DMF and MeOH, but stabilisation of $H P^{-}$is absent in DMSO $(\triangle S \approx 0) \cdot{ }^{[12]} \mathrm{So}_{\mathrm{pK}}$ of $\mathrm{H}_{2} \mathrm{TBP}$ and $\mathrm{H}_{2} \mathrm{TAP}$ in $D M F$ are lower than in DMSO. Substituents have considerable electronic influence on the NH-acid centers of porphyrin. The introduction of electron-acceptor substituent $(\mathrm{Cl})$ into the phenyl rings of $\mathrm{H}_{2}$ TPP increases acidity in DMSO by 1-1.3 order of magnitude, while tetra-meso-propyl substitution in $\mathrm{H}_{2} \mathrm{P}$ decreases acidity by 1.5 orders (Table 2). Basic properties of porphyrins in different solvents were also studied systematically. ${ }^{[20-28]}$ Solvation has important role in stabilization of protonated forms. Thermodynamic parameters obtained for dissociation of the dicationic forms of porphyrins evidence, that acid ionization of $\mathrm{H}_{4} \mathrm{P}^{2+}$ leads to the increase of solvation $(\Delta S>0)$. Polar solvents (DMSO, DMF, MeOH) have a good solvation capacity for cationic forms of porphyrins by means of specific solvation of $\mathrm{NH}$-groups. $\Delta S$ value is increased in the the process of dissociatoin of protonated forms. ${ }^{\text {[1-33] }}$ In acetonitrile and nitrobenzene specific solvation of the reaction center is decreased (Table 3). The constants of dissociation of protonated forms $\mathrm{H}_{2} P$ are in accordance with the electronic nature of substituents. Protonation lead to distortion of the planar structure of the porphyrin molecule. Unlike porphyrins tetraazaporphyrins are not protonated on inner $N$-atoms. The thermodynamic compensation effect of energetic investments on ionization by solvation has the linear character (Figure 3). It shows, that energy required for ionization of the $N-H$ bonds is compensated by solvation.
\end{abstract}

Keywords: Porphyrins, acid-base properties, constants of acid ionisation, non-aqueous solvents.

Андрианов Владимир Геннадьевич, родился в 1948 г. В 1971-1974 г2. обучался в аспирантуре Ивановского химико-технологического института (ИХТИ, в наст. время ИГХТУ) под руководством Берёзина Б.Д. В 1974 г. защитил диссер-тациюю на соискание ученой степени к.х.н. по теме "Исследование ионизации порфиринов по кислотному типу», а в 1994 г. - докторскую диссертацию на тему "Структура и кислотно-основные свойства порфиринов". В настоящее время является профессором кафедры органической химии ИГХТУ. Область научных интересов - кислотноосновные и комплексообразующие свойства порфиринов в неводных растворителях.

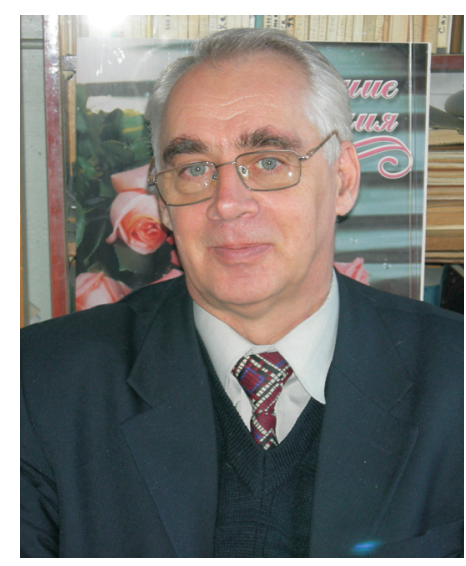

Prof. Andrianov Vladimir Gennad'evich was born in 1948. In 1971-1974 he was post-graduate student at Ivanovo Institute of Chemistry and Technology under supervision of Prof. B.D. Berezin. In 1976 he received the degree "Candidate of Chemical Sciences" (PhD) presenting the dissertation "Investigation of the porphyrin ionization by acidic type", and in 1994 he became Doctor of Chemical Sciences presenting the work "Structure and acid-base properties of porphyrins". Currently he is professor of the Organic Chemistry Department of Ivanovo State University of Chemistry and Technology. The field of his scientific interests includes the acid-base and complex-formation properties of porphyrins in nonaqueous solutions. 


\title{
Кислотно-основные свойства порфиринов в неводных растворителях
}

\author{
В.Г. Андрианов, О.В. Малкова \\ Ивановский государственный химико-технологический университет, Иваново, 153000, Россия \\ E-mail:shukhto@isuct.ru
}

\begin{abstract}
К исследованию кислотно-основных свойств в Ивановском химико-технологическом университете (в те годы в химико- технологическом институте) приступили в коние шестидесятых годов двадиатого века. Полученные впервые количественные характеристики кислотно-основных взаимодействий позволили сделать значительный вклад в область исследований химии порфиринов. В данной работе проведен анализ части полученных в эти годы результатов и подведены некоторые итоги работы по изучению кислотноосновных свойств порфиринов.
\end{abstract}

Ключевые слова: Порфирины, кислотно-основные свойства, константы кислотной ионизации, неводные растворители.

Кислотно-основные свойства являются одними из наиболее важных физико-химических свойств порфиринов. ${ }^{[1-3]}$ В случае сложных кислот и оснований процесс передачи протона является многостадийным и может протекать через ряд промежуточных кислотноосновных форм. ${ }^{[4,5]}$ Поэтому особую роль для развития современной теории кислотно-основного взаимодействия представляют исследования специфики многоцентровых кислот и оснований таких, как порфирины $\left(\mathrm{H}_{2} \mathrm{P}\right)$ и их структурные аналоги. ${ }^{[6,7]}$ Понятие кислотноосновных свойств тетрапиррольных соединений охватывает образование анионных и катионных форм кислотно-основной природы, протекающее с изменением заряда за счет протонного обмена в основной или кислой средах.

Порфирины можно рассматривать как типичные амфотерные соединения, обладающие одновременно основными (N-основания) и очень слабыми кислотными (NН-кислоты) свойствами. В зависимости от кислотности среды можно получить как одно-, так и двухзарядные ионы, находящиеся в равновесии друг с другом и с нейтральной формой.

В первом приближении (без участия растворителя и стабилизации образующихся частиц противоионами) процессы кислотно-основного взаимодействия порфи- ринов могут быть описаны уравнениями (1-4):

$$
\begin{aligned}
& \mathrm{H}_{4} \mathrm{P}^{2+} \stackrel{\boldsymbol{K}_{1}}{\rightleftarrows} \mathrm{H}^{+}+\mathrm{H}_{3} \mathrm{P}^{+} \\
& \mathrm{H}_{3} \mathrm{P}^{+} \stackrel{\boldsymbol{K}_{2}}{\rightleftarrows} \mathrm{H}^{+}+\mathrm{H}_{2} \mathrm{P} \\
& \mathrm{H}_{2} \mathrm{P} \stackrel{\boldsymbol{K}_{3}}{\rightleftarrows} \mathrm{H}^{+}+\mathrm{HP} \\
& \mathrm{HP}^{-} \stackrel{\boldsymbol{K}_{4}}{\rightleftarrows} \mathrm{H}^{+}+\mathrm{P}^{2-}
\end{aligned}
$$

Порфирины способны отдавать сильному основанию и присоединять в кислой среде по два протона (имеется в виду протонирование пирролениновых (=N-) и депротонирование пиррольных (-NH-) атомов азота в центре молекулы), образуя «предельные» катионные $\left(\mathrm{H}_{4} \mathrm{P}^{2+}\right)$ и анионные $\left(\mathrm{P}^{2-}\right)$ формы. Равновесия 1, 2 устанавливаются в полярных электронодонорных средах (метаноле, ацетонитриле, диметилформамиде). Дианионные формы образуются в растворе по всей вероятности только в особо жестких условиях.

Из-за взаимозависимости кислотно-основных центров, входящих в единую сопряженную $\pi$-систему порфирина, ${ }^{[7]}$ следовало бы ожидать изменения основных свойств в противоположном направлении по сравнению с кислотными, однако в действитель-
Малкова Ольга Васильевна, родилась в 1957 году, в 1985-1988 г2. обучалась в аспирантуре Ивановского химико-технологического института (ИХТИ, в наст. время ИГХТУ) под руководством Березина Б.Д. В 1988 2. Защитила диссертацию на соискание ученой степени к.х.н. на тему «Исследование основной ионизации синтетических порфиринов в ацетонитриле”. В настоящее время является дочентом кафедры органической химии ИГХТУ. Область научных интересов - кислотно-основные и комплексообразующие свойства порфиринов в неводных растворителях.

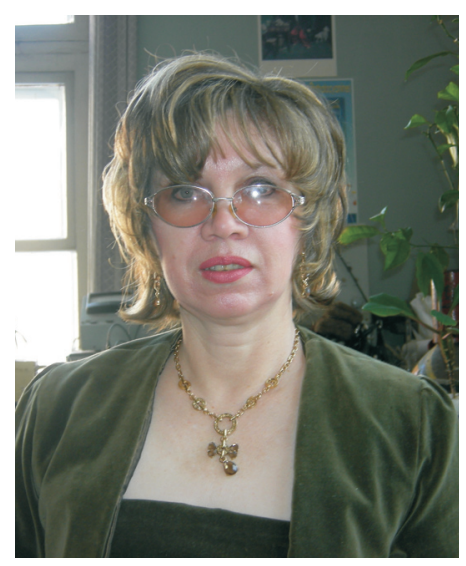

Dr. Malkova Ol'ga Vasil'evna was born in 1957. During 1985-1988 she studied at the post-graduate course of Ivanovo Institute of Che-mistry and Technology under supervision of Prof. B.D. Berezin and Dr. V. G. Andrianov. In 1988 she received the degree "Candidate of Chemical Sciences" (PhD) presenting the dissertation "The study of basic ionization of synthetic porphyrins in acetonitrile". Currently she has the position of associate professor in the Organic Chemistry Department at Ivanovo State University of Chemistry and Technology. The field of her scientific interests includes acid-base and complex-formation properties of porphyrins in non-aqueous solutions. 


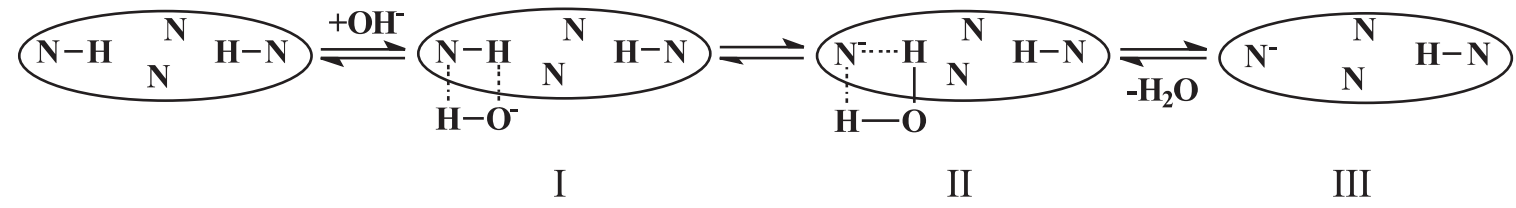

Схема 1.

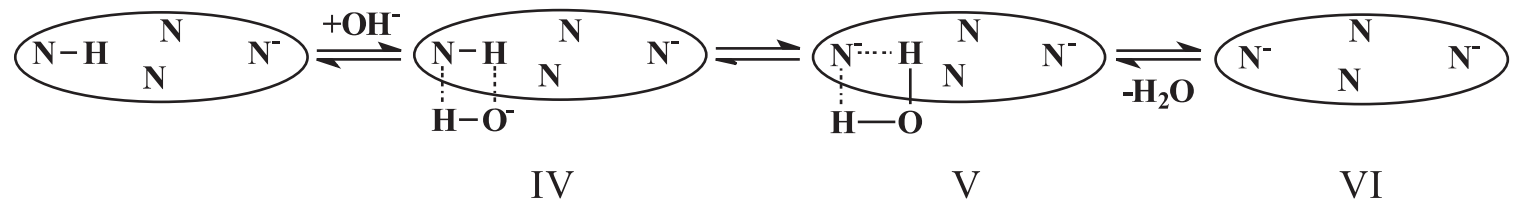

Схема 2.

ности этого не происходит. Причина состоит в том, что устойчивость кислотно-основных форм зависит от ряда факторов, а влияние их специфично для каждой из частиц. Как в случае кислотной, так и основной ионизации порфиринов, факторы стабилизации ионных частиц будут одинаковы, отличаясь лишь по величине вклада в общую стабилизацию, а также по характеру действия.

Различают электронные, сольватационные и стерические факторы стабилизации ионных форм порфиринов кислотно-основного типа в растворах..$^{[8-9]}$

Первая группа факторов характеризуется изменением состояния электронной плотности макрокольца в процессе протонирования или депротонирования, в том числе и при активном участии электронов $\beta$-, мезои (или) $N$-заместителей молекулы $\mathrm{H}_{2} \mathrm{P}$.

Сольватационные факторы обусловлены стабилизацией ионных форм $\mathrm{H}_{2} \mathrm{P}$ в растворе за счет молекул растворителя (Solv), а также ассоциативных связей с противоионами $\mathrm{M}^{+}$или $\mathrm{X}^{-}$.

Стерические факторы стабилизации включают способность ионной частицы к искажению (если это необходимо по структурным соображениям).

При действии на порфирины неорганических щелочей в полярных растворителях (например, $\mathrm{MeOH}$ ) взаимодействие чаще ограничивается образованием ионно-молекулярных ассоциатов $\quad \mathrm{N}-\mathrm{H}^{\cdots} \mathrm{OH}^{-} \quad(\mathbf{I})^{[6]}$ (Схема 1).

Полная ионизация связи N-H (III) возможна у порфиринов только при воздействии очень активных основных реагентов, например, гидроокиси тетраалкиламмония $\mathrm{R}_{4} \mathrm{~N}^{+} \mathrm{OH}^{-}$, или высоких концентраций алкоголят-ионов, или некоторых других сильных оснований органической природы. ${ }^{[10-11]}$

Отрицательный заряд моноанионной формы делокализуется по координационному центру (V), что не благоприятствует более глубокой, двукратной ионизации в силу различных причин, в том числе снижения протонизации второй связи N-H. Концентрации находящихся в равновесии ионных форм резко уменьшаются в ряду $\mathbf{I V}>\mathbf{V}>\mathbf{V} \mathbf{I}^{[12]}$ (Схема 2$)$.

Такая ситуация наблюдается при кислотной ионизации порфиринов, исследованных методом растворимости в метаноле. ${ }^{[6,12-13]}$ Константы ионизации природных и синтетических порфиринов, полученные методом растворимости, представлены в Таблице 1.
Полученные в работах ${ }^{[13,14]}$ величины $\mathrm{p} K_{3}$ позволяют выстроить следующий ряд соединений по увеличению кислотных свойств (Таблица 1$): \mathrm{H}_{2} \mathrm{TPP}\left(p-\mathrm{OCH}_{3}\right)$ $<\mathrm{H}_{2}$ TPP $<\mathrm{H}_{2} \mathrm{DP}<\mathrm{H}_{2} \mathrm{PP}<\mathrm{H}_{2} \mathrm{EP}<\mathrm{H}_{2} \mathrm{MP}<\mathrm{H}_{2} \mathrm{Chl}(\mathrm{a})$ $<\mathrm{H}_{2} \mathrm{Chl}(\mathrm{b})$. Тот факт, что хлорофилловая кислота (a) является более сильной, чем хлорофилловая кислота (b), объясняется, скорее всего тем, что на атомах азота в $\mathrm{H}_{2} \mathrm{Chl}(\mathrm{b})$ электронная плотность выше, следствием чего является пониженная протонизация связи $\mathrm{N}-\mathrm{H}$, т.е. ее большая ковалентность.

Методом спектрофотометрического титрования с потенциометричеким определением кислотности среды было проведено исследование кислотной диссоциации ряда порфиринов в среде диметилсульфоксида. ${ }^{[15]}$

В Таблице 2 представлены величины $K_{3}$ и термодинамические параметры равновесия образования моноанионов порфиринов в ДМСО (вторая ступень ионизации в эксперименте не наблюдается), полученные при титровании растворов порфиринов гидроокисью тетрабутиламмония спектропотенциометрическим методом. Спектральные изменения $\mathrm{H}_{2} \mathrm{TPP}$ при титровании его раствором гидроокиси в ДМСО, а также кривые титрования приведены на Рисунке 1. Из значений $\Delta S$ видно, что в этой среде вклад сольватации в общее изменение энергии Гиббса $\Delta G$ невелик (процессы практически изоэнтропийны), поэтому можно считать, что величины $\mathrm{p} K_{3}$ достоверно отражают влияние электронных эффектов заместителей в молекулах порфиринов на их кислотные свойства.

Стабильность моноаниона в среде ДМСО $\left[\mathrm{N}\left(\mathrm{CH}_{3}\right)_{4}\right] \mathrm{OH}$ сильно возрастает по мере роста жесткости (ароматичности) тетрапиррольной молекулы: порфин < $\mathrm{H}_{2}$ TВP $<\mathrm{H}_{2} \mathrm{TAP}<\mathrm{H}_{2} \mathrm{Pc}\left(\mathrm{SO}_{3}^{-}\right)$. В ДМСО кислота тетрабензопорфин по сравнению с порфином сильнее на 4 порядка, тетраазапорфин - на 10, а моносульфокислота фталоцианина - более чем на 12 порядков. Как показывает опыт, ${ }^{[16-17]}$ кислотность связей N-H азапорфиринов характеризуется сильной температурной зависимостью. В ДМСО и ДМФА число отщепляющихся протонов зависит от температуры. Так, при 318 К в ДМСО можно наблюдать образование дианиона моносульфокислоты фталоцианина. Для $\mathrm{H}_{2}$ ТАР в среде ДМСО характерно ступенчатое отщепление $\mathrm{NH}$-протонов, причем $\mathrm{p} K_{3}$ возрастает с ростом температуры в 2,5 раза быстрее по сравнению с $\mathrm{p} K_{4}$, что приводит к нивелированию констант и их полному равенству при $283,5 \mathrm{~K}\left(\mathrm{p} K_{3}=\mathrm{p} K_{4}=14,5\right) .^{[18]}$ 


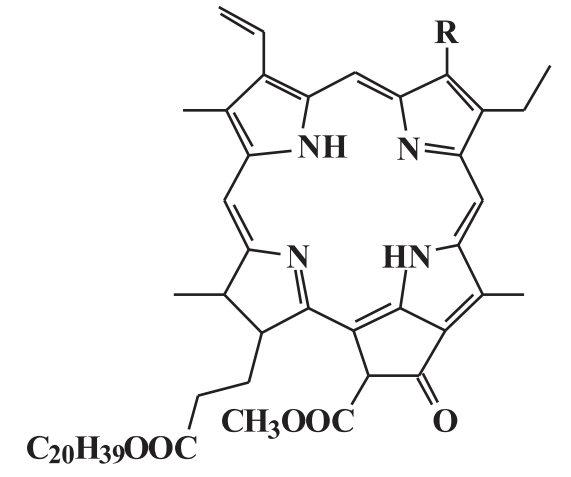

1: $\mathrm{R}=\mathrm{Me}($ Хл(a))

2: $\mathrm{R}=\mathrm{CHO}$

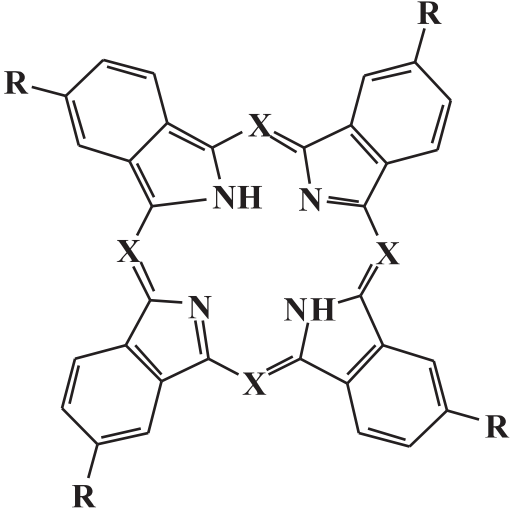

11: $\mathrm{X}=\mathrm{CH}, \mathrm{R}=\mathrm{H}$

12: $\mathrm{X}=\mathrm{N}, \mathrm{R}=\mathrm{H}$

13: $X=N, R=\mathrm{SO}_{3} \mathrm{H}$

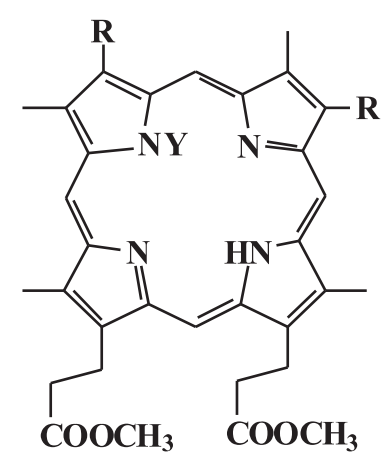

3: $\mathrm{Y}=\mathrm{H}, \mathrm{R}=\mathrm{H}$

4: $\mathrm{Y}=\mathrm{H}, \mathrm{R}=\mathrm{Et}$

5: $\mathrm{Y}=\mathrm{H}, \mathrm{R}=\mathrm{Vi}$

6: $\mathrm{Y}=\mathrm{H}, \mathrm{R}=\mathrm{CH}(\mathrm{OH}) \mathrm{CH}_{3}$

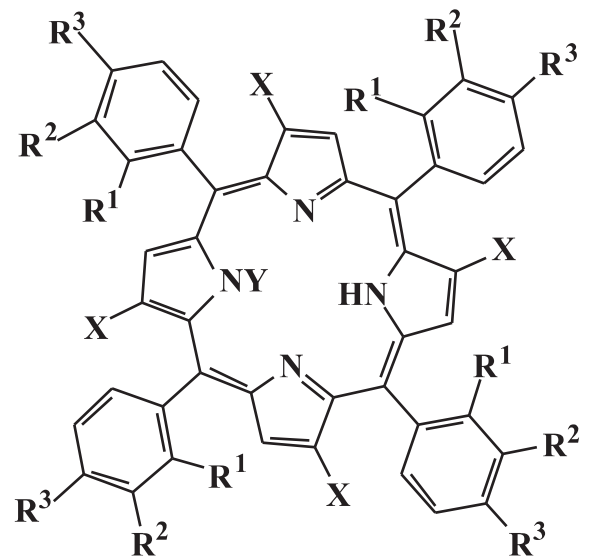

7: $\mathrm{R}^{1}=\mathrm{R}^{2}=\mathrm{R}^{3}=\mathrm{X}=\mathrm{Y}=\mathrm{H}$

8: $R^{1}=R^{2}=X=Y=H, R^{3}=O M e$

14: $R^{2}=R^{3}=X=Y=H, \quad R^{1}=B r$

15: $R^{1}=R^{3}=X=Y=H, \quad R^{2}=B r$

16: $R^{1}=R^{2}=X=Y=H, R^{3}=B r$

17: $\mathrm{R}^{1}=\mathrm{R}^{2}=\mathrm{R}^{3}=\mathrm{Y}=\mathrm{H}, \mathrm{X}=\mathrm{Br}$

18: $R^{2}=R^{3}=X=Y=H, \quad R^{1}=F$

Таблица 1. Константы ионизации $\left(K^{298}\right)$ и термодинамические параметры кислотной ионизации порфиринов в метаноле. ${ }^{[14]}$

\begin{tabular}{|c|c|c|c|c|}
\hline Порфирин & $K^{298}$ & $\Delta H$, кДж/моль & $\Delta G$, кДж/моль & $\Delta S$, кДж/моль·К \\
\hline $\mathrm{H}_{2} \mathrm{Chl}(\mathrm{a}), \mathbf{1}$ & $2,3 \cdot 10^{-15}$ & 40,2 & 83,4 & -145 \\
\hline $\mathrm{H}_{2} \mathrm{Chl}(\mathrm{b}), 2$ & $1,3 \cdot 10^{-15}$ & 36,4 & 85,1 & -163 \\
\hline $\mathrm{H}_{2} \mathrm{MP} \mathrm{д.м.э.,} 3$ & $7,1 \cdot 10^{-16}$ & 28,1 & 86,3 & -195 \\
\hline $\mathrm{H}_{2} \mathrm{EP}, 4$ & $1,6 \cdot 10^{-16}$ & 16,8 & 90,1 & -246 \\
\hline $\mathrm{H}_{2} \mathrm{PP}$ д.м.э., 5 & $2,9 \cdot 10^{-16}$ & 12,5 & 88,6 & -255 \\
\hline $\mathrm{H}_{2} \mathrm{DP}$ д.м.э., 6 & $3,2 \cdot 10^{-16}$ & 41,8 & 88,2 & -156 \\
\hline $\mathrm{H}_{2} \mathrm{TPP}, 7$ & $4,5 \cdot 10^{-17}$ & 12,2 & 89,3 & -273 \\
\hline $\mathrm{H}_{2} \mathrm{TPP}\left(p-\mathrm{OCH}_{3}\right), \mathbf{8}$ & $3,6 \cdot 10^{-17}$ & 10,5 & 93,9 & -280 \\
\hline
\end{tabular}

Таблица 2. Кислотные свойства порфиринов в системе ДМСО - $\left[\mathrm{N}\left(\mathrm{CH}_{3}\right)_{4}\right] \mathrm{OH} .{ }^{[15]}$

\begin{tabular}{ccccc}
\hline Порфирин & $\mathrm{p}_{3}^{298}$ & $\Delta G$, кДж/моль & $\Delta H$, кДж/моль & $T \cdot \Delta S$, кДж/моль \\
\hline $\mathrm{H}_{2} \mathrm{DP}, \mathbf{6}$ & 25,3 & 144,4 & - & - \\
$\mathrm{H}_{2} \mathrm{~T}(n-\mathrm{Pr}) \mathrm{P}, \mathbf{9}$ & $23,91 \pm 0,02$ & $134 \pm 1,0$ & $111 \pm 5$ & $-25 \pm 5$ \\
$\mathrm{H}_{2} \mathrm{P}, \mathbf{1 0}$ & $22,35 \pm 0,02$ & $127,5 \pm 0,1$ & $121 \pm 1$ & $-7 \pm 1$ \\
$\mathrm{H}_{2} \mathrm{TBP}, \mathbf{1 1}$ & $18,53 \pm 0,05$ & $105,7 \pm 1,2$ & $82 \pm 8$ & $-23 \pm 9$ \\
$\mathrm{H}_{2} \mathrm{TAP}, \mathbf{1 2}$ & $12,36 \pm 0,18$ & $70 \pm 1$ & $232 \pm 6$ & $162 \pm 7$ \\
$\mathrm{H}_{2} \mathrm{Pc}\left(\mathrm{SO}_{3}^{-}\right), \mathbf{1 3}$ & $10,73 \pm 0,03$ & $61,2 \pm 0,2$ & $70 \pm 6$ & $-8 \pm 6$ \\
\hline
\end{tabular}


Добавление в раствор моноаниона порфирина значительных количеств хлоридов щелочных металлов приводит к образованию ионных ассоциатов $\mathrm{M}_{2}^{+} \mathrm{P}^{2-}$ вместо ожидаемых НР-. Состав соли подтверждается характером кривых титрования и тангенсом угла наклона зависимостей $\lg \operatorname{Ind}=f(\mathrm{p} H)$, определяющим число протонов, отщепляющихся в процессе титрования порфирина. Показано, ${ }^{[18,19]}$ что при титровании порфиринов раствором $\mathrm{NaOH}$ в ДМСО в присутствии $0,013 \mathrm{M} \mathrm{KCl} \operatorname{tg} \alpha=2$.

Конформационно более жесткие $\mathrm{H}_{2} \mathrm{Pc}$ и $\mathrm{H}_{2}$ ТАР не дают ионных солей с катионами щелочных металлов. ${ }^{[15]} \mathrm{H}_{2}$ ТВР образует их только с $\mathrm{Li}^{+}$, что указывает на снижение необходимости в стабилизации анионов этих порфиринов катионами металлов $\mathrm{M}^{+}$, а также на наличие стерических помех их образованию. Таким образом, при увеличении склонности порфиринового лиганда к ионизации связи NH необходимость в стабилизации аниона отпадает. ${ }^{[4]}$

Моноанионы двухосновных порфиринов ионных ассоциатов с катионами щелочных металлов не образуют. Такие соли обнаружены только на примере $\mathrm{N}$-алкилированных одноосновных порфиринов, например, для $\mathrm{H}(\mathrm{N}-\mathrm{Me}) \mathrm{TPP}$.

Природа растворителя оказывает значительное влияние на кислотные свойства порфиринов. Его роль в процессе диссоциации состоит в сольватации образующихся ионов - продуктов диссоциации. Эффект растворителя особенно проявляется в случае порфиринов с неклассическими свойствами. Некоторые порфиразины, а также пространственно напряженные порфирины, ${ }^{[19]}$ способны давать молекулярные комплексы с переносом протона на молекулу электронодонорного растворителя (алифатический амин, Ру, ДМФА, ДМСО), образование которых фиксируется в ЭСП. В ДМСО дополнительная стабилизация НР- растворителем почти не наблюдается $(\Delta S \approx 0)$, чего нельзя сказать о ДМФА или МеОН. ${ }^{[15]}$ По-видимому, именно с этим связан некоторый рост кислотных свойств порфиринов в ДМФА по сравнению с ДМСО. ${ }^{[12]}$ Так, $K_{3}$ $\mathrm{H}_{2}$ ТВР и $\mathrm{H}_{2}$ ТАР составили в ДМФА, соответственно, 12,0 и 9,68 .
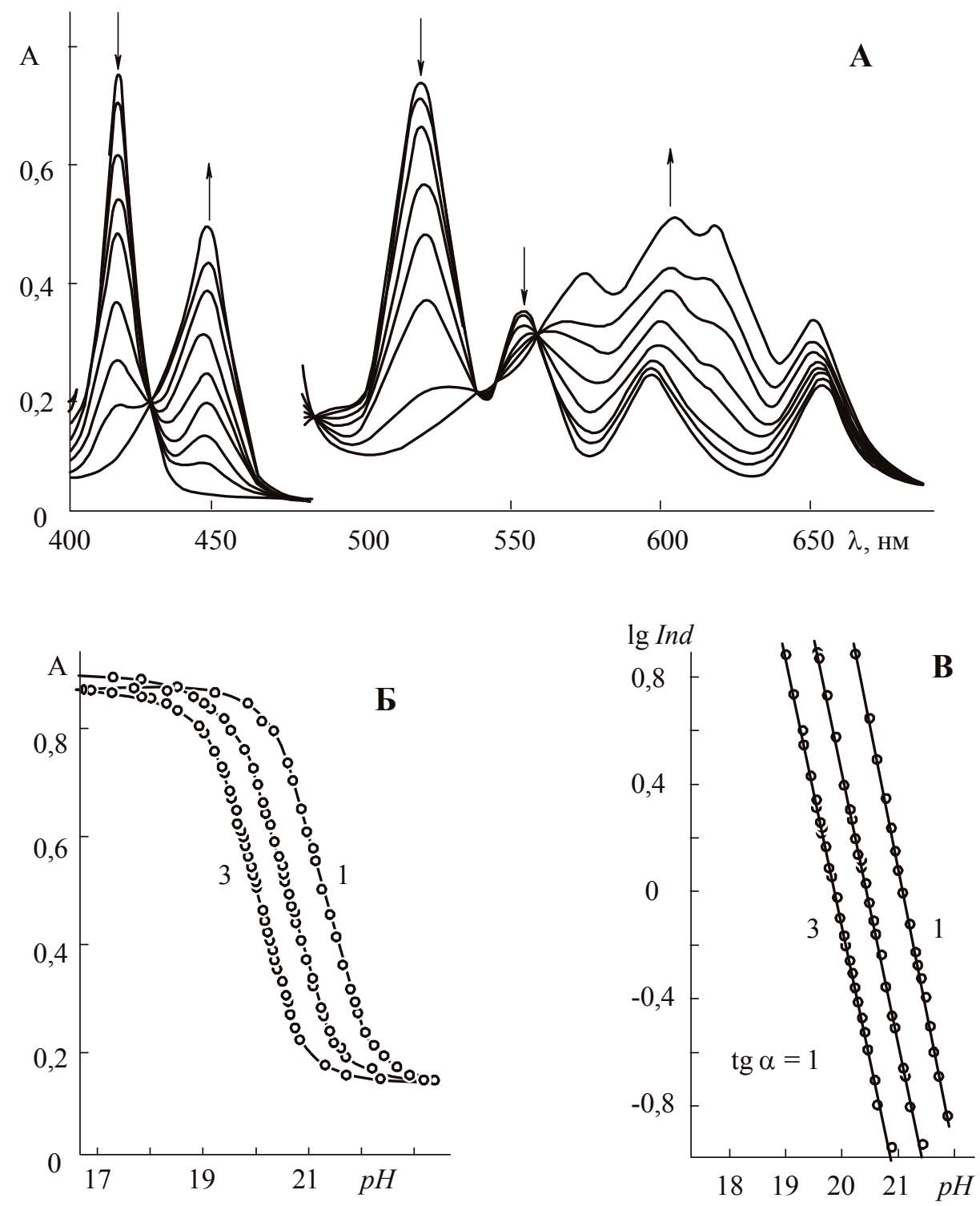

Рисунок 1. Изменение ЭСП тетрафенилпорфина в ДМСО в интервале $\mathrm{p} H 19$ - 22 (А), кривые титрования (Б) и соответствующие им зависимости $\lg \operatorname{Ind}=f(\mathrm{pH})(\mathrm{B}): 1-298,2-308,3-318$ К. ${ }^{[19]}$ 
Классические порфирины к передаче протона на Solv не способны, поскольку их N-Н связи локализованы. В то же время показано, ${ }^{[19]}$ что стабилизация анионных форм классических порфиринов катионом металла является также важным фактором, как и стабилизация растворителем. Титрование $\mathrm{H}_{2}$ ТРР гидроокисью калия на фоне $0,013 \mathrm{M} \mathrm{KCl} \mathrm{в} \mathrm{ДМСО} \mathrm{ведет,} \mathrm{минуя} \mathrm{моно-анион,}$ к образованию дикалиевой соли порфирина $\mathrm{K}_{2}{ }^{+} \mathrm{P}^{2-} \cdot{ }^{[18]}$ Дикалиевая соль порфирина более стабильна, чем его моноанион, так как существует в области меньших значений $\mathrm{p} H .^{[15]}$ Установлено, что в случае соли дианиона $\mathrm{M}_{2}^{+} \mathrm{P}^{2-}$, сходной с дикатионом по симметрии и структуре ЭСП, эти аномалии не воспроизводятся, ${ }^{[10,19]}$ что позволяет предположить отсутствие в необходимости искажения плоской структуры порфирина при кислотной ионизации. Аргументом в пользу данного утверждения является и тот факт, что замещенный по внутрициклическому атому азота $N$-метилтетрафенилпорфин (H(N-Me)TPP), непланарная структура которого доказана, ${ }^{[21]}$ обладает менее выраженными кислотными свойствами (примерно на порядок в ДМСО $\left.{ }^{[12]}\right)$ по сравнению с плоским Н $\mathrm{H}_{2}$ ТР (Таблица 2). Это означает, что электронодонорный эффект группы $\mathrm{CH}_{3}(+\mathrm{I})$, введенной непосредственно в координационный центр молекулы порфирина, приводящий к деполяризации NH-связи превалирует над стерическим эффектом искажения в процессе кислотной ионизации. На планарность анионных форм порфиринов указывает и рост их кислотных свойств по мере усиления ароматичности. ${ }^{[16]}$ Проявление другого важного структурного фактора макроциклического эффекта (МЦЭ) ${ }^{[22]}$ - способствует процессу кислотной диссоциации, несмотря на то, что одна из его составляющих - структурная, отвечающая за экранирование реакционного центра, препятствует удалению NH-протона. В то же время основное действие оказывает электронная составляющая МЦЭ, определяющая легкость и эффективность передачи электронных эффектов в пределах макрокольца и возрастающая по мере усиления жесткости молекулы.

Электронное влияние заместителей эффективно передается на атомы азота кислотных центров порфиринов независимо от того, находится ли заместитель у атома азота, в $\beta$-, непосредственно в мезо- или в одном из положений мезо-фенильного кольца. Так, введение в фенильные кольца $\mathrm{H}_{2}$ ТРР электроноакцепторных атомов хлора, проявляющих преимущественно -I-эффект, приводит к росту кислотности в ДМСО на $1-1,3$ порядка в зависимости от их расположения, ${ }^{[19]}$ тогда как тетра-мезо-пропильное замещение снижает кислотность связи NH на 1,5 порядка по сравнению с порфином (Таблица 1). Очевидно, это результат общего влияния искажения и электронодонорного мезо-заместителя. Наиболее эффективным оказалось $\beta$-алкильное замещение молекулы дейтеропорфирина, оно понижает кислотные свойства порфина почти на 3 единицы $\mathrm{p} H$.

Систематические исследования основных свойств порфиринов различных структурных групп в среде растворителей различной донорно-акцепторной природы были проведены в наших работах спектрофотопотенциометрическим методом. ${ }^{[23-31]}$

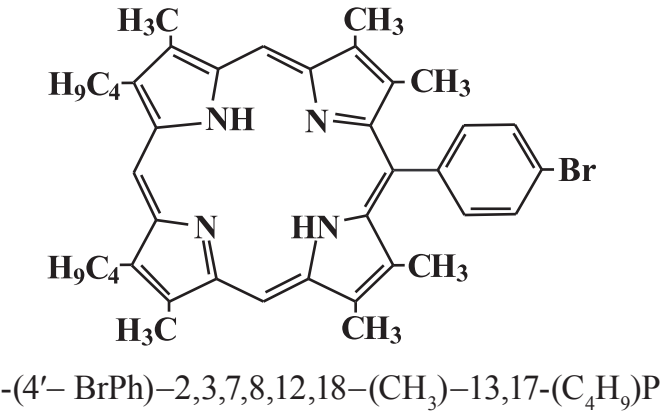

Природа растворителя определяет состояние катионной формы порфирина - форма, стабилизированная растворителем, или форма, вступающая в ассоциативные равновесия с противоионами. В результате нарушения планарности катионных форм доступ молекул растворителя и других реакционноспособных частиц к их реакционным центрам облегчается. При этом особую роль в стабилизации частиц приобретает сольватация. ${ }^{[25-27,31]}$ По сравнению с плоским нейтральным порфирином, где макроцикл стабилизирован в растворе в основном за счет сил универсальной сольватации, ${ }^{[33]}$ при сольватации дикатиона большую роль играют специфические взаимодействия. Из термодинамических параметров диссоциации также видно, что процесс удаления двух протонов дикатиона $\mathrm{H}_{4} \mathrm{P}^{2+}$ в массу растворителя и освобождение более плоских молекул $\mathrm{H}_{2} \mathrm{P}$ сопровождается ростом числа частиц в системе и десольватацией в процессе диссоциации (как правило $\Delta S>0)$. Практически во всех случаях при диссоциации катионных форм порфиринов наблюдается термодинамический компенсационный эффект. ${ }^{[4]}$ Он выражается в линейной зависимости в координатах $\Delta H=f(\Delta S)$, которая показывает, что энергетические затраты на разрыв связи N-Н компенсируются за счет энтропийной составляющей, а для процесса, протекающего в растворе - за счет сольватации ионных частиц.

Протон, вступая во взаимодействие с порфирином, образует с его основным $\mathrm{N}$-атомом ковалентную $\sigma$-связь со значительной долей ионности. В сущности, соединения, образованные путем координации протона лигандом, могут быть отнесены к классу координационных, а сам протон определен, как координирующая частица вследствие родственности акцепторных свойств иона металла и протона. Полярные координирующие растворители (ДМСО, ДМФА, МеОН) эффективно сольватируют катионные формы порфиринов за счет возрастающего вклада специфической сольватации NH-групп. Об этом свидетельствуют положительные значения $\Delta S$ процесса их диссоциации (Таблицы 2, 3) во всех изученных растворителях за исключением АН, слабо сольватирующего как катионные, так и анионные частицы. ${ }^{[34-37]}$ В то же время глубина протонирования (глубина внедрения протона в электронную оболочку донорного атома ${ }^{[4,37]}$ в этих растворителях достаточно низка, поскольку протоны в растворе первоначально сильно связываются растворителем и лишь затем передаются на реакционный центр. Противоположная картина наблюдается в растворителях с меньшим значением протонного сродства (например, ацетонитрил, 

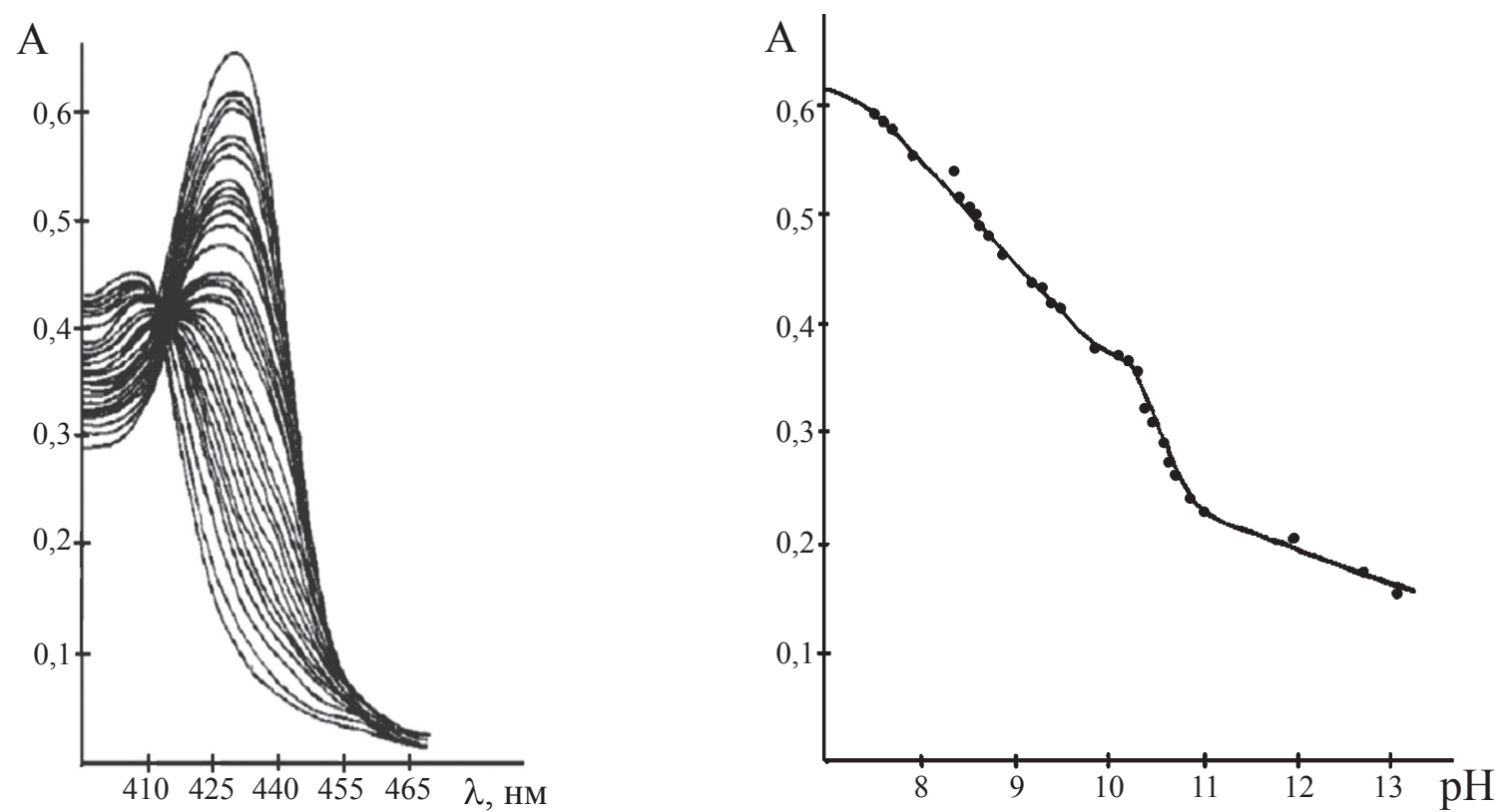

Рисунок 2. Изменения электронного спектра поглощения соединения и кривая титрования для 5-(4'- $\mathrm{BrPh})-2,3,7,8,12,18-\left(\mathrm{CH}_{3}\right)$ -13,17-( $\left.\mathrm{C}_{4} \mathrm{H}_{9}\right)$ Р при увеличении кислотности раствора (ацетонитрил-хлорная кислота), 298 К. ${ }^{[31]}$

нитробензол), где выше степень передачи протона на реакционный центр, а значит и выше глубина протонирования порфирина; степень специфической сольватации на NH-центрах снижается (Таблица 3).

Природа растворителя не является единственной причиной фиксируемости $\mathrm{H}_{3} \mathrm{P}^{+}$В ЭСП и на кривых титрования. Данные ${ }^{[25]}$ показывают, что стабильность монокатиона возрастает в том случае, если плоская структура исходного порфирина нарушена. Это наблюдается у сильно искаженных $N$-замещенных порфиринов. ${ }^{[20,29]}$ Спектрально фиксируемое ступен-чатое присоединение протонов (Таблица 3) имеет место и в случае тетра$2,7,12,17-б р о м-5,10,15,20$-тетрафенилпорфирина. ${ }^{[27]}$ Известно, что введение большого числа заместителей в $\beta$-положения молекулы $\mathrm{H}_{2} \mathrm{TPP}$, приводящее в пределе к додеказамещению, делает структуру молекулы сильно искаженной. ${ }^{[39]}$

Протонирование приводит к искажению плоской структуры молекулы и нарушению как электронной, так и стерической составляющих МЦЭ.. ${ }^{[22]}$ В результате МЦЭ дикатионов порфиринов более напоминает МЦЭ нежестких макроциклов. По мере того, как структура $\mathrm{H}_{2} \mathrm{P}$ становится более жесткой, процесс присоединения протонов по внутрициклическим атомам азота осложняется, а основные свойства молекул понижаются. Так, более ароматичный и не склонный к деформациям по сравнению с собственно порфиринами $\mathrm{H}_{2}$ ТВР менее устойчив в кислой среде, а его напряженный монокатион уже при 318 К разрушается в растворе АН с деструкцией макроцикла. ${ }^{[25]}$ Дальнейший рост ароматичности молекулы при переходе к тетразапорфиринам приводит к тому, что большинство соединений этого класса по внутрициклическим атомам вообще не протонируются, присоединяя протон к одному из мезо-атомов азота. ${ }^{[4,10]}$ Структурная компонента МЦЭ препятствует процессу присоединения протона в центр молекулы.

Основность порфиринов коррелирует с природой заместителей, расположенных на периферии молекулы. Реакции диссоциации протонированных форм порфиринов не являются изоэнтропийными, как для соединений различных структурных групп, так и в различных растворителях, поэтому по величинам $\mathrm{p} K$ невозможно однозначно судить об изменении прочности $\mathrm{N}-\mathrm{H}$ связей под влиянием заместителя. ${ }^{[12]}$ Тем не менее, константы диссоциации протонированных форм замещенных $\mathrm{H}_{2} \mathrm{P}$ находятся в хорошем согласии с классическими представлениями о природе заместителей, даже тех, которые введены не непосредственно в макроцикл, а через фенильный «буфер». Буферный эффект мезофенильных заместителей соединений играет положительную роль в процессе делокализации избыточного, как положительного, так и отрицательного заряда, и приводит, в частности, к возрастанию как кислотных, так и основных свойств тетрафенилпорфиринов по сравнению с незамещенным порфином (Таблицы 1, 2). Впрочем, рост основных свойств $\mathrm{H}_{2}$ ТРР объясня-

Таблица 3. Влияние природы растворителя на суммарное равновесие диссоциации $\mathrm{H}_{4}$ TPP $^{2+}$.[23-25,38]

\begin{tabular}{cccccc}
\multicolumn{5}{c}{$\mathrm{H}_{4} \mathrm{P}^{2+} \stackrel{\boldsymbol{K}_{1}}{\longleftarrow} 2 \mathrm{H}^{+}+\mathrm{H}_{2} \mathrm{P}$} \\
\hline Растворитель & $\mathrm{DN}$ & $\mathrm{p} K_{1,2}^{298}$ & $\Delta G$, кДж/моль & $\Delta H$, кДж/моль & $\Delta S$, Дж/(моль К) \\
\hline $\mathrm{AН}$ & 14,1 & $19,8 \pm 0,2$ & $113 \pm 1$ & $86 \pm 6$ & $-43 \pm 10$ \\
$\mathrm{MеОН}$ & 19,0 & $7,50 \pm 0,10$ & $43 \pm 2$ & $69 \pm 4$ & $85 \pm 16$ \\
ДМФА & 26,6 & $6,14 \pm 0,04$ & $35 \pm 1$ & $94 \pm 2$ & $198 \pm 7$ \\
ДМСО & 29,8 & $3,20 \pm 0,02$ & $18,4 \pm 1,0$ & $30 \pm 3$ & $57 \pm 6$ \\
\hline
\end{tabular}


Таблица 4. Термодинамические параметры диссоциации протонированных форм порфиринов в среде $\mathrm{AH}_{-} \mathrm{HClO}_{4} \cdot{ }^{[25-27]}$

\begin{tabular}{ccccc}
\hline Порфирин & \multicolumn{2}{c}{$p K^{298}$} & $\Delta$ Н, кДж/моль & $\Delta S$, Дж/(моль К $)$ \\
\hline $\mathrm{H}_{2} \mathrm{P}, \mathbf{1 0}$ & $\mathrm{p} K_{1}$ & $6,20 \pm 0,15$ & $41 \pm 1$ & $20 \pm 2$ \\
& $\mathrm{p} K_{2}$ & $9,15 \pm 0,15$ & $105 \pm 1$ & $178 \pm 17$ \\
$\mathrm{H}_{2} \mathrm{TPP}, \mathbf{7}$ & $\mathrm{p} K_{1,2}$ & $15,35 \pm 0,15$ & $146 \pm 2$ & $-43 \pm 10$ \\
$\mathrm{H}_{2} \mathrm{TPP}(o-\mathrm{Br})_{4}, \mathbf{1 4}$ & $\mathrm{p} K_{1,2}$ & $19,8 \pm 0,2$ & $86 \pm 6$ & $-42 \pm 23$ \\
& $\mathrm{p} K_{1}$ & $6,78 \pm 0,01$ & $27 \pm 10$ & $-77 \pm 33$ \\
& $\mathrm{p} K_{2}$ & $9,15 \pm 0,02$ & $29 \pm 16$ & $-114 \pm 3$ \\
$\mathrm{H}_{2} \mathrm{TPP}(\boldsymbol{M}-\mathrm{Br})_{4}, \mathbf{1 5}$ & $\mathrm{p} K_{1,2}$ & $15,93 \pm 0,02$ & $56 \pm 18$ & $70 \pm 7$ \\
$\mathrm{H}_{2} \mathrm{TPP}(p-\mathrm{Br})_{4}, \mathbf{1 6}$ & $\mathrm{p} K_{1,2}$ & $17,68 \pm 0,08$ & $120 \pm 15$ & $-174 \pm 10$ \\
$\mathrm{H}_{2} \mathrm{TPP}(2,7,12,17-\mathrm{Br})_{4}, \mathbf{1 7}$ & $\mathrm{p} K_{1,2}$ & $17,10 \pm 0,05$ & $51 \pm 17$ & $111 \pm 11$ \\
& $\mathrm{p} K_{1}$ & $6,42 \pm 0,05$ & $70 \pm 5$ & $-279 \pm 28$ \\
& $\mathrm{p} K_{2}$ & $7,54 \pm 0,05$ & $-36 \pm 4$ & $154 \pm 41$ \\
$\mathrm{H}_{2} \mathrm{TPP}(o-\mathrm{F})_{4}, \mathbf{1 8}$ & $\mathrm{p} K_{1,2}$ & $13,98 \pm 0,10$ & $34 \pm 9$ & $-17 \pm 8$ \\
& $\mathrm{p} K_{1}$ & $6,98 \pm 0,08$ & $35 \pm 1$ & $130 \pm 10$ \\
& $\mathrm{p} K_{2}$ & $8,80 \pm 0,08$ & $89 \pm 3$ & $113 \pm 18$ \\
\hline
\end{tabular}

Таблица 5. Влияние природы заместителей $(\mathrm{X})$ мезо-фенильных групп $\mathrm{H}_{2} \mathrm{TPP}(\mathrm{X})_{4}$ на устойчивость дикатионов $\mathrm{H}_{4} \mathrm{P}^{2+}$ в системе органический растворитель - хлорная кислота. ${ }^{[15,25-27]}$

\begin{tabular}{|c|c|c|c|c|c|c|}
\hline \multirow{2}{*}{ Заместитель } & \multicolumn{3}{|c|}{ ДМСО } & \multicolumn{3}{|c|}{$\mathrm{AH}$} \\
\hline & $\mathrm{p} K_{1,2}$ & $\Delta H$, кДж/моль & $\Delta S$, Дж/(моль·К) & $\mathrm{p} K_{1,2}$ & $\Delta H$, кДж/моль & $\Delta S$, Дж/(моль·К) \\
\hline$p-\mathrm{OCH}_{3}$ & $5,14 \pm 0,03$ & $51,1 \pm 1,7$ & $65 \pm 5$ & $21,10 \pm 0,10$ & $114 \pm 11$ & $-20 \pm 2$ \\
\hline$p-\mathrm{CH}_{3}$ & $4,84 \pm 0,05$ & $31 \pm 3$ & $57 \pm 6$ & $22,06 \pm 0,10$ & $74 \pm 4$ & $-174 \pm 12$ \\
\hline $\mathrm{H}$ & $3,20 \pm 0,02$ & $37,3 \pm 1,7$ & $63 \pm 6$ & $19,8 \pm 0,2$ & $86 \pm 6$ & $-43 \pm 10$ \\
\hline$p$-Br & - & - & - & $17,92 \pm 0,04$ & $51 \pm 10$ & $-170 \pm 30$ \\
\hline$p-\mathrm{SO}_{3}^{-}$ & - & - & - & $16,7 \pm 0,2$ & 83 & -44 \\
\hline$p-\mathrm{NO}_{2}$ & $2,36 \pm 0,06$ & $18 \pm 2$ & $52 \pm 5$ & $15,96 \pm 0,10$ & $58 \pm 6$ & $-111 \pm 10$ \\
\hline
\end{tabular}

ется также большей склонностью этого макроцикла к деформациям.

Введение атомов $\mathrm{Br}$, проявляющих по отношению к бензольному фрагменту индукционный $(-\mathrm{I})$ и слабый $+\mathrm{C}$-эффект сопряжения, в пара-положения фенильных колец $\mathrm{H}_{2}$ ТРР (Таблицы 4, 5) приводит к ослаблению основных свойств молекулы. Это связано с преобладанием -I-эффекта атома $\mathrm{Br}$. Введение сульфо- и нитро- групп в пара-положения фенильных колец еще больше понижает основные свойства этих порфиринов. Напротив, введение в молекулу $\mathrm{H}_{2}$ ТРР электронодонорных групп - $\mathrm{CH}_{3}$ и - $\mathrm{OCH}_{3}$ заметно увеличивает основность, причем $\mathrm{H}_{2} \mathrm{TPP}\left(p-\mathrm{OCH}_{3}\right)_{4}$, в котором заместитель обладает значительным + С-эффектом, намного более основен по сравнению с $\mathrm{H}_{2} \mathrm{TPP}\left(p-\mathrm{CH}_{3}\right)_{4}{ }^{\left[{ }^{[26]}\right.}$

Таким образом, исследование процессов кислотной диссоциации протонированных форм порфиринов в растворах в присутствии хлорной кислоты показывает, что наличие электронодонорных или электроноакцепторных групп на периферии молекулы влияет на состояние электронной плотности на внутрициклических атомах азота. Нитро-группы и протонированные амино-группы понижают электронную плотность на пирролениновых атомах азота вследствие действия отрицательного эффекта сопряжения, что приводит к ослаблению связи азот - водород и увеличению кислотных свойств протонированных форм порфириновых молекул. Введение объемных заместителей в положения макрокольца частично искажает плоскость ароматического ядра макрокольца и, как следствие, в меньшей степени влияет на состояние электронной плотности внутрициклических атомов азота.

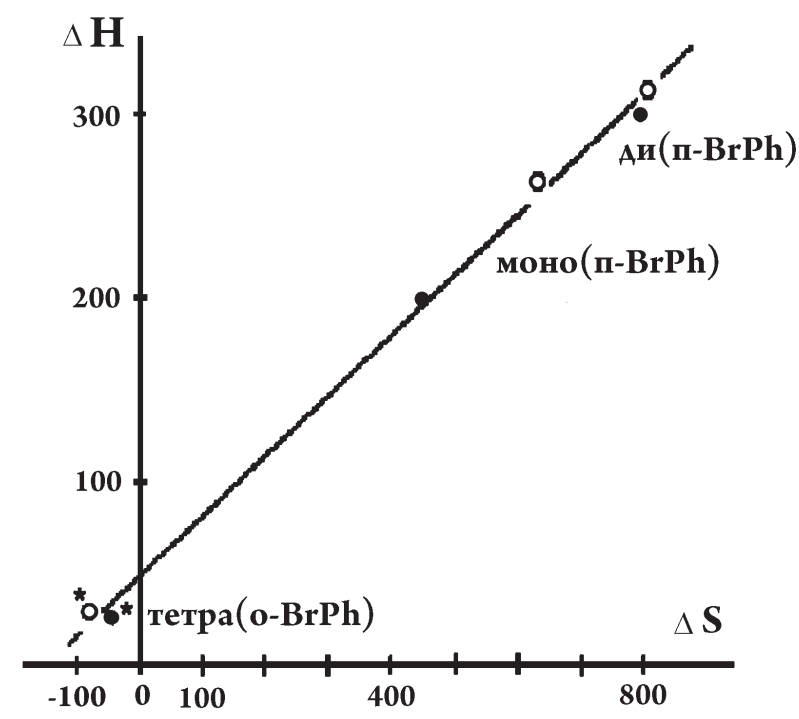

Рисунок 3. Сольвато-термодинамический эффект кислотной диссоциации протонированных форм порфиринов: $\bullet-\mathrm{p} K_{3}$; о - $K_{4} ; *$ - литературные данные..$^{[25-27]}$ 
Практически во всех случаях при диссоциации катионных форм порфиринов наблюдается термодинамический компенсационный эффект (Рисунок 3). ${ }^{[4]} \mathrm{OH}$ выражается в линейной зависимости в координатах $\Delta H$ $=f(\Delta S)$, которая показывает, что энергетические затраты на разрыв связи NH компенсируются за счет энтропийной составляющей, а для процесса, протекающего в растворе - за счет сольватации ионных частиц.

\section{Список литературы}

\section{References}

1. Bell R. Proton v Khimii [Proton in Chemistry] M.: Mir, 1977, 382 p. (in Russ).

2. Albert A., Serjent E. Konstanti Ionizatsii Kislot i Osnovanii [Ionization Constants of Acids and Bases] M.: Khimia, 1964, 180 p. (in Russ).

3. Fialkov Y.I. Rol' Rastvoritelya $v$ Tekhnologicheskom Prossesse [The Role of the Solvent in the Technological Process] L.: Khimia, 1990, 238 p. (in Russ).

4. Berezin B.D. Koordinatsionnye Soedinenia Porfirinov $i$ Ftalotsianinov [Coordination Compounds of Porphyrins and Phthalocyanines] M.: Nauka, 1978, 280 p. (in Russ).

5. Izmailov N.A. Elektrokhimia Rastvorov [Electrochemisty of Solurions] M.: Khimia, 1976, 488 p. (in Russ).

6. Berezin B.D. Andrianov V.G. Izv. Vyssh. Ucheb. Zaved., Khim. Khim. Tehnol. 1977, 20, 807-816 (in Russ).

7. Berezin B.D. Andrianov V.G. Malkova O.V. Dokl. Akad. Nauk SSSR 1990, 314, 1432-1435 (in Russ).

8. Malkova O.V., Andrianov V.G., Berezin B.D. Zh. Fiz. Khim. 1995, 69, 1588-1591 (in Russ).

9. Berezin D.B. Vliyanie Elektronnykh, Solvatatsionnikh $i$ Stericheskikh Faktorov na Khromofornye Svoistva Porfirinov $i$ ikh Protonirovannykh Form [Influence of Electronic, Solvation and Steric Factors on the Chromofore Properies of the Porphyrins and their Protonated Forms], Dis. kand. khim. nauk, Ivanovo, ISUCT, 1997, 187 p.. (in Russ).

10. Richards R.A., Hammonds K., Joe M., Miscelly G.M. Inorg. Chem. 1996, 35, 1940-1944.

11. Schroeder G., Brzezinski B., Podebski D., Grech E. J. Molec. Struct. 1997, 416, 11-19.

12. Andrianov V.G. Struktura i Kislotno-Osnovnye Svoistva Porfirinov [Structure and the Acid-Base Properties of Porphyrins], Diss. dok. khim. nauk, Ivanovo, ICNR RAN, 1994, 273 p. (in Russ).

13. Berezin B.D., Koifman O.I., Andrianov V.G. Zh. Fiz. Khim. 1973, 47, 1464-1466 (in Russ).

14. Sheinin V.B., Andrianov V.G., Berezin B.D. Izv. Vyssh. Ucheb. Zaved., Khim. Khim. Tehnol. 1981, 24, 964-969 (in Russ.).

15. Sheinin V.B. Issledovanie Kislotno-Osnovnoi Ionizatsii Nekotorykh Prirodnykh $i$ Sinteticheskikh Porphirinov [Investigation of Acid-Base Ionization of Some Natural and Synthetic Porphyrins], Dis. kand. khim. nauk, Ivanovo, ICTI, 1981, 176 p. (in Russ).

16. Sheinin V.B., Andrianov V.G., Berezin B.D., Koroleva T.A. Zh. Org. Khim. 1985, 21, 1564-1570 (in Russ).
17. Sheinin V.B., Berezin B.D., Khelevina O.G., Stuzhin P.A., Telegin F.Y. Zh. Org. Khim. 1985, 21, 1571-1575 (in Russ).

18. Andrianov V.G., Sheinin V.B., Berezin B.D. In: Tez. III Vses. Konf. po Khimii. i Biokhimii Porfirinov [Abstract Book of the III' Conference on Chemistry and Biochenistry of Porphyrins] Samarkand, 1982, 77 (in Russ).

19. Sheinin V.B., Andrianov V.G., Berezin B.D. Zh. Org. Khim. 1984, 20, 2192-2197 (in Russ).

20. Berezin B.D., Berezin D.B. Sostoyanie Khimii Neklassicheskikh Porfirinov [The Chemistry of Nonclassic Porphyrins] In: Uspekhi Khimii Porfirinov [Advances in Porphyrin Chemistry] (Golubchikov O.A., Ed.) SPb.: NII Khimii, 1999, 2, 128-141 (in Russ).

21. Lavallee D.K. Chemistry and Biochemistry of N-Substituted Porphyrins. New-York: VHC Publishers, 1987, 313 p.

22. Lomova T.V., Berezin D.B. Makrotsiklicheskiy Effekt Metalloporfirinov [Macrocyclic Effect of Metal Porphyrins] In: Problemy Khimii Rastvorov [The Problems of Solution Chemistry] (Kutepov A.M., Ed.) M.: Nauka, 2001, 326-362 (in Russ).

23. Andrianov V.G., Milyukova N.P., Berezin B.D. Zh. Fiz. Khim. 1985, 59, 2891-2893 (in Russ).

24. Andrianov V.G., Milyukova N.P. Berezin B.D. Izv. Vyssh Ucheb. Zaved., Khim. Khim. Tekhnol. 1986, 29(1), 112-115 (in Russ).

25. Malkova O.V., Berezin B.D. Andrianov V.G. Izv. Vyssh. Ucheb. Zaved., Khim. Khim. Tekhnol. 1986, 29(1), $37-39$ (in Russ).

26. Malkova O.V., Andrianov V.G., Berezin B.D. Izv. Vyssh. Ucheb. Zaved., Khim. Khim. Tekhnol. 1988, 31(11), 36-39 (in Russ).

27. Malkova O.V., Andrianov V.G., Berezin B.D., Semeikin A.S., Zhilina G.I. Zh. Org. Khim. 1988, 24, 1546-1550 (in Russ).

28. Shushin S.A., Andrianov V.G., Berezin B.D. Zh. Fiz. Khim. 1990, 64, 1243-1247 (in Russ.).

29. Shushin S.A., Andrianov V.G., Berezin B.D. Izv. Vyssh. Ucheb. Zaved., Khim. Khim. Tehnol. 1988, 31(6), 53-57 (in Russ).

30. Andrianov V.G., Malkova O.V., Loyko V.E., Berezin B.D. Zh Fiz. Khim. 1994, 68, 366-367 (in Russ).

31. Malkova O.V., Bazlova I.Yu., Andrianov V.G., Semeikin A.S., Berezin B.D. Zh. Fiz. Khim. 1997, 71, 1432-1435 (in Russ).

32. Berezin D.B., Semeikin A.S., Andrianov V.G., Berezin M.B. Zh. Org. Khim. 2000, 70, 1541-1546. (in Russ.).

33. Vjugin A.I., Krestov G.A. Termodinamika Protsessov Rastvoreniya i Solvatatsii Prirodnykh $i$ Sinteticheskykh Porfirinov i ikh Kompleksov [Thermodinamics of Dissolution and Solvation Processes of Natural and Synthetic Porphyrins and their Complexes] M.: Nauka, 1989, 137-179 (in Russ).

34. Kreshkov A.P. Analiticheskaya Khimiya $v$ Nevodnikh Rastvorakh [Analytical Chemistry in Non-Aqueous Solutions] M.: Khimiya, 1982, 256 p. (in Russ).

35. Reimers J.R., Hall L.E. J. Amer. Chem. Soc. 1999, 121, 37303744.

36. Pashin I.V., Sheinin V.B., Berezin B.D. Izv. Vyssh. Ucheb. Zaved., Khim. Khim. Tekhnol. 2000, 43(4), 125-130.

37. Berezin B.D. Zh. Obshch. Khim. 1973, 43, 2738-2743 (in Russ).

38. Sheinin V.B., Andrianov V.G., Berezin B.D. Izv. Vyssh. Ucheb. Zaved., Khim. Khim. Tekhnol. 1981, 24, 47-50 (in Russ).

39. Kalisch W., Senge M.O. Tetrahedron Letters 1996, 37, 11831186. 